\title{
Anisotropic cosmological models with MacCallum parameter
}

\author{
Purnachandra Rao Koya \\ School of Mathematical \& Statistical Sciences, Hawassa University, P. O. Box - 5, Hawassa, Ethiopia \\ Email address: \\ drkpraocecc@yahoo.co.in
}

To cite this article:

Purnachandra Rao Koya. Anisotropic Cosmological Models with MacCallum Parameter. American Journal of Astronomy and Astrophysics. Vol. 2, No. 1, 2014, pp. 1-5. doi: 10.11648/j.ajaa.20140201.11

\begin{abstract}
In this paper, we have presented two anisotropic cosmological models, of which the former being T-model is homogeneous and the latter being non T-model is inhomogeneous. We have constructed formula for all the physical and kinematical quantities and established relations among them. Equations of state are constructed. Both these solutions can be applied to all the epochs of the universe for which $\xi \epsilon[0,1)-\{1 / 2\}$, where the quantity $\xi$ is a MacCallum parameter and describes the anisotropy of the 4-dimensional space-time. It is explicitly shown that the T-model presented here is more general solution in the sense that it includes the one given by McVitte and Wilt-shire.
\end{abstract}

Keywords: Cosmological Models, Anisotropy, Homogeneity, MacCallum Parameter

\section{Introduction}

It is well known that the Friedmann-Robertson-Walker (F-R-W) cosmological models, derived on the twin assumptions of spatial isotropy and homogeneity, provided a satisfactory description of the observable universe for a considerable part of its entire history. However, the existence of inhomogeneities in the form of galaxies and clusters as well as the observed anisotropy in the spectrum of the cosmic background radiation could not be explained with the help of F-R-W models. Cosmological models with inhomogeneous density have been studied by $[1,2,3,4]$ and others. These models as well as the models given in [5] provide certain generalizations of the F-R-W models. The Tolman, Bondi as well as Kantowski and Sachs models are anisotropic.

In order to associate a cosmological model to certain epoch of the universe, one has to compare the strength of the free gravitational field $\boldsymbol{\varepsilon}$ with that of the matter field $\boldsymbol{\rho}$. The strong relativistic cases are $\varepsilon>\rho$ in general and $\varepsilon=2 \rho$ in particular. The expression defined by [6] in terms of shear tensor $\sigma_{a}^{b}$ and expansion $\theta$ of the material time-like congruence works as a supplement to penrose's hypothesis and that a physically meaningful cosmological model should evolve in such a way that the quantity $\xi$ starts from 0 at the "big bang" and ends up with 1 in the "black hole" (viz. big crunch). Inhomogeneous Kantowski-Sacs type cosmological models with $\xi=0$ have been studied in
[7]. Spherically symmetric cosmological models with $\xi=1$ (highly chaotic state) have been investigated by [8]. Shearing T-models with $\xi=1 / 2$ have been studied in [9] and shown that the pressure isotropy $p_{r}=p_{\perp}$ implies $\varepsilon=2 \rho$ and vice versa. Cosmological solutions, other than t-models, with $\xi=1 / 2$ and with non-vanishing conformal Weyl tensor are studied in [10] and shown that shear does not vanish. The existence of Event and Particle horizons in these models has been studied by [11, 12] and given the graphical representations. In the present paper we present two cosmological solutions which are applicable to all epochs of the universe for which $\xi \in[0,1)-\{1 / 2\}$.

\section{Spherically Symmetric Material Distribution}

It is well known that the geometry of the most general spherically symmetric space-time is described by the metric

$$
d s^{2}=-\exp (\lambda) d r^{2}-R^{2}\left(d \theta^{2}+\sin ^{2} \phi\right)+\exp (v) d t^{2}
$$

where $\lambda, R$ and $v$ are functions of $\mathrm{r}$ and $\mathrm{t}$ only. Further, $R$ and $\mathrm{r}$ are called respectively the Eulerian (or positive) and Lagrangian (or labeling) coordinates; i.e. $R(r, 0)=r$. We now assume that the matter filling the space-time given by the metric (1) is an anisotropic fluid, as [13]

$$
T_{a}^{b}=\left(\rho+p_{\perp}\right) u_{a} u^{b}-p_{\perp} g_{a}^{b}+\left(p_{r}-p_{\perp}\right) v_{a} v^{b}
$$


where $p_{r}$ and $p_{\perp}$ being respectively radial and transverse fluid pressures and

$$
\begin{aligned}
& u^{a}=(0,0,0, \exp (-v / 2)), u_{a} u^{a}=1, \\
& v^{a}=(\exp (-\lambda / 2), 0,0,0), v_{a} v^{a}=1,
\end{aligned}
$$

The Einstein field equations

$$
-8 \pi T_{a}^{b}=R_{a}^{b}-(1 / 2) R g_{a}^{b}+\wedge g_{a}^{b}
$$

after using (1) to (4) in (5), gives the following:

$$
\begin{gathered}
8 \pi T_{1}^{1}+\Lambda=-8 \pi p_{r}+\Lambda=-[1 / R \exp (\lambda)]\left[\left(R^{\prime 2} / R\right)+R^{\prime} v^{\prime}\right]+[1 / R \exp (v)]\left[2 \ddot{R}+\left(\dot{R}^{2} / R\right)-\dot{R} \dot{v}\right]+\left[1 / R^{2}\right] \\
8 \pi T_{2}^{2}+\Lambda=8 \pi T_{3}^{3}+\Lambda=-8 \pi p_{\perp}+\Lambda=8 \pi \varepsilon-[1 / R \exp (\lambda)]\left[2 R^{\prime \prime}-\left({R^{\prime}}^{2} / R\right)-R^{\prime} \lambda^{\prime}+R^{\prime} v^{\prime}\right]+[1 / R \exp (v)]\left[2 \ddot{R}-\left(\dot{R}^{2} / R\right)+\dot{R} \dot{\lambda}-\dot{R} \dot{v}\right]-\left[1 / R^{2}\right] \\
8 \pi T_{4}^{4}+\Lambda=8 \pi \rho+\Lambda=-[1 / R \exp (\lambda)]\left[2 R^{\prime \prime}+\left({R^{\prime}}^{2} / R\right)-R^{\prime} \lambda^{\prime}\right]+[1 / R \exp (v)]\left[\left(\dot{R}^{2} / R\right)+\dot{R} \dot{\lambda}\right]+\left[1 / R^{2}\right] \\
8 \pi T_{4}^{1}=0=[1 / R \exp (\lambda)]\left[2 \dot{R}^{\prime}-R^{\prime} \dot{\lambda}-\dot{R} v^{\prime}\right]
\end{gathered}
$$

$$
\begin{array}{r}
8 \pi \varepsilon=\exp (-\lambda)\left[\left(R^{\prime \prime} / R\right)-\left(R^{\prime} / R\right)^{2}-\left(v^{\prime \prime} / 2\right)-\left(v^{\prime 2} / 4\right)-\left(R^{\prime} \lambda^{\prime} / 2 R\right)+\left(R^{\prime} v^{\prime} / 2 R\right)+\left(\lambda^{\prime} v^{\prime} / 4\right)\right]+\exp (-v)[(\ddot{\lambda} / 2)+ \\
\left.\left(\dot{\lambda}^{2} / 4\right)-(\ddot{R} / R)+(\dot{R} / R)^{2}-(\dot{R} \dot{\lambda} / 2 R)+(\dot{R} \dot{v} / 2 R)-(\dot{\lambda} \dot{v} / 4)\right]+\left(1 / R^{2}\right)
\end{array}
$$

Here $\varepsilon$ is the eigenvalue of the conformal Weyl tensor in Petrov's classification [14]. Here and in what follows a prime and an overhead dot for $\lambda, R$ and $v$ denote respectively a differentiation with respect to $r$ and $t$. Since, the eigenvalue $\varepsilon$ is always coupled with the material energy density $\rho$, the former is interpreted as the "energy density of the free gravitational field" and its presence is related with both anisotropy and inhomogeneity [15-20]. The necessary and sufficient condition for conformal flatness of the space-time (1) is $\varepsilon=0$. Using the

$$
e^{-\lambda}{R^{\prime 2}}^{2}=\Gamma^{2}=1+U^{2}-(8 \pi / 3)\left(\rho+\varepsilon+p_{\perp}-p_{r}+(\Lambda / 8 \pi)\right) R^{2}
$$

We now directly write down the equations which govern the evolution of the system as below [13]:

$$
\begin{gathered}
D_{t}(\lambda / 2)=\partial U / \partial R \\
D_{t} \Gamma=U D_{r}(v / 2) \\
D_{t}\left(n R^{2}\right)=-n R^{2}(\partial U / \partial R) \\
D_{t} U=(1 / 2) \Gamma^{2}(\partial U / \partial R)-(4 \pi / 3)\left[\rho+\varepsilon+p_{\perp}+2 p_{r}-(\Lambda / 4 \pi)\right] R \\
D_{t}\left\{(4 \pi / 3)\left[\rho+\varepsilon+p_{\perp}-p_{r}+(\Lambda / 8 \pi)\right] R^{3}\right\}=-\left(p_{r}-(\Lambda / 8 \pi)\right) D_{t}\left[(4 \pi / 3) R^{3}\right] \\
D_{r}\left\{(4 \pi / 3)\left[\rho+\varepsilon+p_{\perp}-p_{r}+(\Lambda / 8 \pi)\right] R^{3}\right\}=\left(\rho+D_{r}\left[(4 \pi / 3) R^{3}\right]\right) D_{r}\left[(4 \pi / 3) R^{3}\right] \\
\left(\rho+p_{\perp}\right) D_{r}(v / 2)=-D_{r}\left[p_{r}-(\Lambda / 8 \pi)\right]+\left(p_{\perp}-p_{r}\right) D_{r}\left\{\ln \left[R^{2} \exp (v / 2)\right]\right\}
\end{gathered}
$$

where $\mathrm{n}$ denotes the baryon number density and we have written $\partial / \partial R=\left(R^{\prime}\right)^{-1}(\partial / \partial r)$.

We may mention here that the energy density of the free gravitational field which is coupled always to the material energy density as well as the cosmological constant plays a significant role in describing truly relativistic situation. We have taken, for simplicity, the coupling constant as unity. However, in order to raise the contribution of $\varepsilon$ substantially relative to $\rho$, we could have chosen the coupling constant to desired levels.

\section{Kinematics of Spherically Symmetric Models}

Spherically symmetric solutions can be classified according to their kinematical properties. The assumption of spherically symmetry implies that rotation $w_{a b}=$ 0 and hence the fluid velocity field must be hyper surface orthogonal. From (1) and (3) we get the expressions for the remaining kinematical quantities, viz. acceleration $\dot{u}_{a}$, components of the shear tensor $\sigma_{a}^{b}$, shear invariant $\sigma$ defined by $\sigma^{2}=(3 / 2) \sigma_{a}^{b} \sigma_{a}^{b}$, and expansion $\theta$ of the time-like congruence $u^{a}$, as below:

$$
\dot{u}_{a}=[\exp (-\lambda / 2)]\left(v^{\prime} / 2\right)
$$




$$
\begin{gathered}
\sigma=[\exp (-v / 2)][(\dot{\lambda} / 2)-(\dot{R} / R)] \\
\theta=\sigma+3(\dot{R} / R)
\end{gathered}
$$

With the help of these expressions, MacCallum (1982) defined the quantity $\xi$ which describe the anisotropy in the space-time as $\xi=\left|3 \sigma_{a}^{b} \sigma_{b}^{a} / 2 \theta^{2}\right|^{1 / 2}$ and that, in our present case, reduces to $\xi=|[(\dot{\lambda} / 2)-(\dot{R} / R)] /[(\dot{\lambda} / 2)+(2 \dot{R} / R)]|=|\sigma / \theta|$ or equivalently it can be written as [13]

$$
(1 \mp \xi)(\dot{\lambda} / 2)=(1 \pm 2 \xi)(\dot{R} / R)
$$

Similarly, the Raychaudhuri [21] equation can be rewritten as

$$
\dot{\theta}=-(1 / 3) \theta^{2}-(2 / 3) \sigma^{2}-4 \pi\left(\rho+p_{r}+2 p_{\perp}\right)+\Lambda
$$

We now introduce a cosmological T-model and derive evolution equations and expressions for the kinematical quantities in the next section. Further we show that this solution is more general in the sense that it includes the one given by McVitte and Wilt-shire.

\section{Cosmological T - Models}

In this section we study T-models. On putting $R^{\prime}=0$, the metric (1) reduces to

$d s^{2}=-\exp (\lambda) d r^{2}-R^{2}(t)\left(d \theta^{2}+\sin ^{2} \phi\right)+\exp (v) d t^{2}$

where $R$ is the function of $t$ only while $\lambda$ and $v$ remain to be the functions of $r$ and $t$.

The space-time described by (27) is an inhomogeneous perturbation of the Kantowski - Sachs [5] metric (1), and is spherically symmetric but does not contain the centers of symmetry in the hyper-surface $t=$ constant . The models described by (27) are called "T-models" in the Russian literature [22-25] for which the standard Schwarzschild coordinates viz. $R(r, 0)=r$, do not exist. For the spacetime metric (27) the Einstein's field equation (9) takes the form $\dot{R} v^{\prime}=0$, and that leads us to consider

$$
\begin{aligned}
& v(r, t)=0 \text { and } \dot{R} \neq 0 \\
& \qquad s^{2}=-\left\{R^{2 n} /\left[1-f^{2}(r)\right]\right\} d r^{2}-R^{2}\left(d \theta^{2}+\sin ^{2} \phi\right)+(\dot{R} / R)^{2} d t^{2}
\end{aligned}
$$
on integrating (25), we get form

where $n=[(1 \pm 2 \xi) /(1 \mp \xi)]$. following form:
Other cases were discussed in the literature [7-10]. Now

$$
\lambda / 2=[(1 \pm \xi) /(1 \mp \xi)] \log R(t)
$$

Thus, on using (28) and (29), the metric (27) takes the

$$
d s^{2}=-R^{2 n}(t) d r^{2}-R^{2}(t)\left(d \theta^{2}+\sin ^{2} \phi\right)+d t^{2}
$$

The expressions for the physical and kinematical quantities, for the space-time metric (30), take the

$$
\begin{gathered}
8 \pi p_{r}-\Lambda=-2(\ddot{R} / R)-(\dot{R} / R)^{2}-\left(1 / R^{2}\right) \\
8 \pi p_{\perp}-\Lambda=-8 \pi \varepsilon-2(\ddot{R} / R)-(2 n-1)(\dot{R} / R)^{2}+\left(1 / R^{2}\right) \\
8 \pi \rho+\Lambda=(2 n+1)(\dot{R} / R)^{2}+\left(1 / R^{2}\right) \\
8 \pi \varepsilon=(n-1)(\ddot{R} / R)+(n-1)^{2}(\dot{R} / R)^{2}+\left(1 / R^{2}\right) \\
\sigma=(n-1)(\dot{R} / R) \\
\theta=\sigma+3(\dot{R} / R)=(n+2)(\dot{R} / R)
\end{gathered}
$$

Here we computed the relationship among $p_{r}, p_{\perp}, \rho$ and $\varepsilon$ and the resultant 'equation of state' is given by

$(2 n+1) \varepsilon=(n-1)[\rho+(\Lambda / 2 \pi)]-(n+2) p_{r}-(2 n-5) p_{\perp}$

Further, we note that the metric (30) reduces to McVitte and Wilt-Shire [26] solution when $R(t)=t$.

\section{General Cosmological Models}

In this section we study general models with $\xi \in[0,1)-$ $\{1 / 2\}$. Thus, on integrating (25) we get $(\lambda / 2)=n \log R-$ $(1 / 2) \log \left[1+f^{2}(r)\right]$ where $n=[(1 \pm 2 \xi) /(1 \mp \xi)]$, and $f(r)$ is an arbitrary function. Also using (25) in (9), we get $(v / 2)=\log \dot{R}-n \log R$. In view of these expressions for $\lambda$ and $v$, the space-time metric (1) reduces

For (38), the expressions for $p_{r}, p_{\perp}, \rho, \varepsilon, a, \sigma$ and $\theta$ are given below:

$$
\begin{aligned}
& 8 \pi p_{r}-\Lambda=\left[R^{\prime}\left(1+f^{2}\right) / R^{2 n+1}\right]\left[\left(2 \dot{R}^{\prime} / \dot{R}\right)-(2 n-1)\left(R^{\prime} / R\right)\right]-(2 n+1) R^{2 n-2}-\left(1 / R^{2}\right) \\
& 8 \pi p_{\perp}-\Lambda=-8 \pi \varepsilon+\left[R^{\prime}\left(1+f^{2}\right) / R^{2 n+1}\right]\left(2 R^{\prime \prime} / R^{\prime}\right)+\left(2 \dot{R}^{\prime} / \dot{R}\right)-(4 n-1)\left(R^{\prime} / R\right)+2\left[f f^{\prime} /\left(1+f^{2}\right)\right]-(4 n-1) R^{2 n-2}+\left(1 / R^{2}\right) \\
& 8 \pi \rho+\Lambda=-\left[R^{\prime}\left(1+f^{2}\right) / R^{2 n+1}\right]\left\{\left(2 R^{\prime \prime} / R^{\prime}\right)-(2 n-1)\left(R^{\prime} / R\right)+2\left[f f^{\prime} /\left(1+f^{2}\right)\right]\right\}+(2 n+1) R^{2 n-2}+\left(1 / R^{2}\right) \\
& 8 \pi \varepsilon=(n+1)\left[R^{\prime}\left(1+f^{2}\right) / R^{2 n+1}\right]\left\{\left(R^{\prime \prime} / R^{\prime}\right)-(2 n+1)\left(R^{\prime} / R\right)+\left[f f^{\prime} /\left(1+f^{2}\right)\right]\right\}+(2 n-1)(n-1) R^{2 n-2}- \\
& {\left[\dot{R}^{\prime}\left(1+f^{2}\right) / R^{2 n} \dot{R}\right]\left\{\left(\dot{R}^{\prime \prime} / \dot{R}^{\prime}\right)-(3 n+1)\left(R^{\prime} / R\right)+\left[f f^{\prime} /\left(1+f^{2}\right)\right]\right\}+\left(1 / R^{2}\right)} \\
& a=\left(\sqrt{1+f^{2}} / R^{\prime}\right)\left[\left(\dot{R}^{\prime} / \dot{R}\right)-n(\dot{R} / R)\right] \\
& \sigma=(n-1) R^{n-1}
\end{aligned}
$$




$$
\theta=\sigma+3 R^{n-1}=(n+2) R^{n-1}
$$

Assuming that the function $R(r, t)$ is separated in its arguments, we can write without loss of generality $R(r, t)=$ $r g(t), g(0)=1$. So that $(25)$ gives $(\lambda / 2)=n \log g(t)-(1 / 2) \log \left[1+f^{2}(r)\right]$ and using this $(\lambda / 2)$ in $(9)$ we get, $(v / 2)=(1-n) \log R$. Hence, in view of these expressions for $\lambda$ and $v$, the space-time metric (1) reduces to

$$
d s^{2}=-\left\{g^{2 n} /\left[1-f^{2}(r)\right]\right\} d r^{2}-r^{2} g^{2}(t)\left(d \theta^{2}+\sin ^{2} \phi\right)+r^{2-2 n} d t^{2}
$$

For (46), the expressions for $p_{r}, p_{\perp}, \rho, \varepsilon, a, \sigma$ and $\theta$ are computed and given below:

$$
\begin{gathered}
8 \pi p_{r}-\Lambda=-\left[\left(1+f^{2}\right) / g^{2 n}\right]\left[(2 n-3) / r^{2}\right]-r^{2 n-2}\left[(2 \ddot{g} / g)+(\dot{g} / g)^{2}\right]-(1 / r g)^{2} \\
8 \pi p_{\perp}-\Lambda=-8 \pi \varepsilon+\left[\left(1+f^{2}\right) / g^{2 n}\right]\left\{\left[(1-2 n) / r^{2}\right]+\left[2 f f^{\prime} / r\left(1+f^{2}\right)\right]\right\}-r^{2 n-2}\left[(2 \ddot{g} / g)+(2 n-1)(\dot{g} / g)^{2}\right]+(1 / r g)^{2} \\
8 \pi \rho+\Lambda=-\left[\left(1+f^{2}\right) / g^{2 n}\right]\left\{\left[1 / r^{2}\right]+\left[2 f f^{\prime} / r\left(1+f^{2}\right)\right]\right\}+r^{2 n-2}(2 n+1)(\dot{g} / g)^{2}+(1 / r g)^{2} \\
8 \pi \varepsilon=-\left[\left(1+f^{2}\right) / g^{2 n}\right]\left\{\left[n^{2} / r^{2}\right]-\left[n f f^{\prime} / r\left(1+f^{2}\right)\right]\right\}+r^{2 n-2}\left[(n-1)(\ddot{g} / g)+(n-1)^{2}(\dot{g} / g)^{2}\right]+(1 / r g)^{2} \\
a=\left(\sqrt{1+f^{2}} / g^{2 n}\right)^{1 / 2}[(1-n) / r] \\
\sigma=(n-1) r^{n-1}(\dot{g} / g) \\
\theta=\sigma+3 r^{n-1}(\dot{g} / g)=(n+2) r^{n-1}(\dot{g} / g)
\end{gathered}
$$

\section{Conclusions}

We have constructed two cosmological solutions to Einstein's field equations. The first one is a T-model and the other is a general model other than T-model. Expressions for the physical quantities $p_{r}, p_{\perp}, \rho$, and $\varepsilon$ are given and the relations, i.e., equations of state, were found. Both the solutions can be applied to all epochs of the universe for which $\xi \in[0,1)-\{1 / 2\}$, where the quantity $\xi$ described the anisotropy of the 4-dimensional spacetime. The T-model presented here is more general solution in the sense that it includes the solution given by McVitte and wilt-shire.

\section{Acknowledgements}

My profound thanks are due to the referee for his encouraging comments and to Mr. Mangamuri Seetha Ramaiah for his support.

\section{References}

[1] R. C. Tolman, Proceedings of the National Academy of Sciences of USA, Vol. 20, 1934, p. 69.

[2] G. C. Omer, the Astrophysical Journal, Vol. 109, 1949, p. 164.

[3] H. Bondi, Monthly Notices of the Royal Astronomical Society, Vol. 107, 1947, p. 410.

[4] W. B. Bonnor, Monthly Notices of the Royal Astronomical Society, Vol. 167, 1974, p. 55.

[5] R. Kantowski and R. K. Sachs', Journal of Mathematical Physics, Vol. 7, 1966, p.443.
[6] M. A. H. MacCallum, In: V. D. Sabbata, ed., the origin and evolution of galaxies, World Scientific Pub. Co., 1982.

[7] J. Krishna Rao, et al., Mathematics Today, Vol. 16, 1998, p. 25.

[8] K. Purnachandra Rao, Mathematics Today, Vol. 17, 1999, p. 29.

[9] K. Purnachandra Rao, Mathematics Today, Vol. 21, 2005, p. 31.

[10] K. Purnachandra Rao, Mathematics Today, Vol. 24, 2008, p. 17.

[11] K. Purnachandra Rao, Mathematics Today, Vol. 27, 2011, p. 54.

[12] K. Purnachandra Rao, Journal of Modern Physics, Vol. 4, 2013 pp. 1194-1199. http://dx.doi.org/10.4236/jmp.2013.49162

[13] J. Krishna Rao, Journal of the Institute of Mathematics, vol. 51, 1995, p. 57.

[14] J. Krishna Rao, Current Science, Vol. 35, 1966, p.389.

[15] J. Krishna Rao, General Relativity and Gravitation, Vol. 2, 1971, pp. 385-386. http://dx.doi.org/10.1007/BF00758157

[16] J. Krishna Rao, Journal of Physics (London), Vol. A5, 1972, p. 479.

[17] J. Krishna Rao, General Relativity and Gravitation, Vol. 2, 1973, p. 351. doi: 10.1007/BF00771004

[18] J. Krishna Rao, Pramana: Journal of Physics, Vol. 34, 1990, p. 423.

[19] J. Krishna Rao and M. Annapurna, Pramana: Journal of Physics, Vol. 27, 1986, p. 637.

[20] J. Krishna Rao and M. Annapurna, Pramana: Journal of 
Physics, Vol. 38, 1992, p. 21.

[21] A. K. Raychaudhri, Physical Review, Vol. 98, 1955, pp. 1123-1126. doi:10.1103/PhysRev.98.1123

[22] I. D. Novikov, Sov. Astron. A. J., Vol. 7, 1964, p.587.

[23] V. A. Ruban, Sov. Phys. JEPT Lett. Vol. 8, 1968, p. 414.
[24] V. A. Ruban, Sov. Phys. JEPT Lett. Vol. 29, 1969, p. 41027.

[25] V. A. Ruban, Sov. Phys. JEPT Lett. Vol. 58, 1983, p. 463.

[26] G. C. McVitte and R. J. Wilt-shire, Int. J. Theor. Phys. Vol. 14, 1975, p. 145. 